\title{
Evaluation of Antibiotic Resistance Pattern of Escherichia coli Isolated From Broiler Chickens With Colibacillosis in Ardabil Province, Iran
}

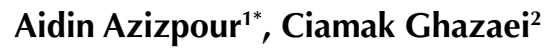 \\ 'Department of Poultry Diseases, University of Mohaghegh Ardabili, Ardabil, Iran \\ 2Department of Microbiology, University of Mohaghegh Ardabili, Ardabil, Iran
}

\author{
*Correspondence to \\ Aidin Azizpour, \\ Tel: +98 045-33512081-9, \\ Fax: +98 045-33510811. \\ Email: Aidin_azizpour@uma.ac.ir
}

Received September 6, 2020

Accepted December 22, 2020

Published online December 30, 2020

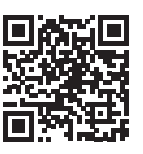

Please cite this article as follows: Azizpour A Ghazaei C. Evaluation of antibiotic resistance pattern of Escherichia coli isolated from broiler chickens with colibacillosis in Ardabil Province, Iran. Int J Basic Sci Med. 2020;5(4):125 130. doi:10.34172/ ijbms.2020.22.

\begin{abstract}
Introduction: Colibacillosis is one of the most important bacterial diseases of birds that is caused by Escherichia coli. This disease causes considerable economic damage to the poultry industry every year. Various antimicrobial agents are used to reduce the damage caused by this infection. But in recent decades, the increased use of antibiotics has led to the development of resistant genes and, consequently increasing antibiotic resistance of bacteria, leading to a decrease in the efficacy of antibiotics. The purpose of this study was to determine the susceptibility and drug resistance of 178 isolates of 40 chicken flocks in Ardabil province northwest of Iran.

Methods: Five carcasses were randomly selected from each flocks with colibacillosis and sampled from liver and heart using sterile swabs. After culture and isolation, colonies were identified by biochemical and serological methods. Antibiotic resistance of all isolates to 19 antibiotics was determined using disk diffusion method based on CLSI guidelines.

Results: Of 200 samples, 178 (89\%) were isolated, and $22(11 \%)$ did not grow. In this study the highest antibiotic resistance was observed against flumequine $(98.31 \%)$, nalidixic acid $(97.25 \%)$, tylosin $(97.20 \%)$, oxytetracycline $(97.20 \%)$, chlortetracycline $(95.50 \%)$, difloxacin $(89.32 \%)$, doxycycline $(81.47 \%)$, enrofloxacin $(77.53 \%)$, sulfamethoxazole + trimethoprim $(71.91 \%)$, and the lowest antibiotic resistance was recorded for Linco-Spectin $(36.52 \%)$, chloramphenicol $(22.47 \%)$, gentamycin $(7.30 \%)$, fuzbac $(5.05 \%)$ and ceftriaxone $(3.93 \%)$. All isolates were highly sensitive to ceftazidime.

Conclusion: The results of this study showed a high level of resistance to antibiotics commonly used in poultry industry, which is probably due to improper use of antibiotics in poultries.

Keywords: Colibacillosis, Antibiotic resistance, Broiler chicken, Isolates
\end{abstract}

\begin{abstract}
Introduction
Escherichia coli is a natural flora of the digestive tract of humans, mammals and birds, which is generally regarded as an opportunistic pathogen and accounts for about $10-15 \%$ of its population as pathogenic strains for birds (APEC; Avian pathogenic Escherichia coli). ${ }^{1,2}$ Often following the damage to the immune system or broken barrier of domestic birds, APEC strains cause systemic or local infections. ${ }^{1,3}$ Colibacillosis is one of the most important bacterial diseases of poultry and turkeys caused by infection with the APEC strains of $E$. coli.
\end{abstract}

It causes septicemia, respiratory tract infections, cellulite, pericarditis, peritonitis, salpingitis, synovitis, colon granulomas, and airbag infections. ${ }^{4,5}$ Noninfectious agents such as stress, exposure to the dust and ammonia inside breeding farms increase the likelihood of damage to the lining cells of the mucosal layer of the respiratory system and cause the invasion of the respiratory system by E. coli. ${ }^{5,6}$ It can also occurs secondary to primary infectious diseases such as infectious bronchitis, Newcastle (including vaccine strains), mycoplasmosis, pasteurellosis, etc., and lead to respiratory complex disease. ${ }^{7}$ However, infection with E. coli is of particular importance and imposes significant economic losses to the poultry industry annually worldwide due to

(c) 2020 The Author(s); Published by Zabol University of Medical Sciences. This is an open-access article distributed under the terms of the Creative Commons Attribution License (http://creativecommons.org/licenses/by/4.0), which permits unrestricted use, distribution, and reproduction in any medium, provided the original work is properly cited. 
increase in mortality and increased carcass removal in the slaughter inspection process. ${ }^{8}$

There are many antimicrobial agents used in poultry industry to control the morbidity and mortality of colibacillosis. In fact, antibiotic therapy is one of the important tools to reduce the incidence and overall damage of bacilli. ${ }^{2,6}$ But in recent years, the overuse of drugs as preventive and therapeutic agents for infections or growth promoters of poultry has led to the emergence and spread of resistant genes and consequently increasing in antibiotic resistance, leading to reduced efficiency has finally made it difficult to treat the disease. ${ }^{9}$ On the other hand, the phenomenon of antibiotic resistance due to the spread of resistant bacteria in human population is also of great importance. ${ }^{8,10,11}$ The aim of this study was to evaluate the frequency of drug resistance of $E$. coli isolates obtained from poultry flocks in Ardabil province against different antibiotics.

\section{Materials and Methods}

\section{Sample Collection}

In this study, 40 broiler flocks (2-6 weeks old) from different parts of Ardebil province with clinical symptoms and suspected autopsy injuries were studied in Mehr Veterinary Laboratory within a year. Five birds were randomly selected from each flock and after sampling and initial diagnosis, the liver and heart were sampled after heating.

\section{Identification of E. coli}

The samples were first cultured in MacConkey agar and incubated at $37^{\circ} \mathrm{C}$ for 24 hours. The lactosepositive colonies were then separated and re-cultured on MacConkey agar until pure culture. ${ }^{9}$ To confirm the bacterial identity, a colony of pure culture was cultured on EMB medium (Eosin methylene blue) and if produced by metallic green glaze, finally was identified and confirmed with biochemical subtraction tests including indole production, methyl red reaction and Voges-Proskauer reaction, urea production, citrate intake, fermentation of glucose, and decarboxylation of ornithine and lysine based on standard bacteriological methods of final diagnosis and confirmation. ${ }^{1,2}$ The E. coli isolates confirmed by biochemical tests were stored in LB medium with 30\% glycerol at $-70^{\circ} \mathrm{C}$ until the test.

\section{Antibiotic Susceptibility Test}

To determine antibiotic resistance pattern of 178 isolates by 19 drugs commonly used in Iranian Poultry Industry was used disc qualitative diffusion method by Kirby Bauer procedure ${ }^{2}$ in accordance with the Clinical and Laboratory Standards Institute (CLSI) guidelines. ${ }^{12}$ For this study, antibiotic discs (in micrograms concentration) sulfamethoxazole + trimethoprim (23.75 / 1.25), amoxicillin (10), ciprofloxacin (5), ceftriaxone (30), tylosin (30), enrofloxacin (5), gentamicin (10), fuzbac
(200), ceftazidime (30), chloramphenicol (30), kanamycin (30), lincospectin (200/15), furazolidone (100), chlortetracycline (30), doxycycline (30), oxytetracycline (30), difloxacin (10), colistin, and nalidixic acid (30) were provided from Padtan Teb Company of Iran.

For disk diffusion experiments, each bacterial isolate was removed from $70^{\circ} \mathrm{C}$ freezer and cultured on MacConkey agar for 24 hours at $37^{\circ} \mathrm{C}$. Then 2 to 3 colonies were transferred from the MacConkey agar medium to the test tube containing TSB (Tryptic Soy Broth) and incubated for 3 hours at $37^{\circ} \mathrm{C}$ to prepare $0.5 \mathrm{McFarland}$ turbidity. Subsequently, sterile swabs from the half-McFarland bacterial suspension were cultured on Müller-Hinton agar medium. After about 10 minutes, antibiotic discs were placed on the inoculated Müller-Hinton medium and plates were incubated at $35^{\circ} \mathrm{C}$ for 18 hours. ${ }^{1,7}$ Then the diameter of the inhibition growth zone of each disk was measured and finally the resistance and sensitivity of each isolate were compared with CLSI standard.

\section{Results}

The results of the present study are shown in Tables 1 to 2 . Out of 200 samples of broilers infected with colibacillosis in Ardebil province, E. coli was isolated in 178 cases $(89 \%)$ and was not grown in 22 cases $(11 \%)$. The results of the disk diffusion test of $E$. coli isolates from cases of broiler poultry colibacillosis in Ardabil province have been presented in Table 1. According to Table 1, 178 E. coli isolates from broiler chickens with colibacillosis were highly susceptible to ceftazidime and the resistance of the isolates to 18 other antibiotics ranged from 3.93\% to $98.31 \%$, which varied in the three resistance groups. In the first group there were 5 antibiotics with low resistance (between 0\%-40\%), chloramphenicol, gentamicin, lincospectin, fosbac and ceftriaxone. The second group of 5 antibiotics colistin, amoxicillin, ciprofloxacin, furazolidone and kanamycin had moderate resistance (between $40 \%$ and $70 \%$ ).

In the third group of 8 antibiotics tylosin, oxytetracycline, chlortetracycline, doxycycline, sulfamethoxazole + trimethoprim, flumequine, difloxacin and nalidixic acid showed high resistance rate (between 70 and 100\%). The isolates were highly resistant (over 95\%) to 5 antibiotics flumequine, tylosin, oxytetracycline, chlortetracycline and nalidixic acid.

Among the resistant isolates, multiple resistance was observed, with $100 \%$ resistant isolates to at least 3 compounds and $0.5 \%$ resistant to at least 18 compounds of the total 19 antibiotics tested (Table 2).

\section{Discussion}

Colibacillosis is one of the most economically important bacterial diseases of poultry industry, especially broilers worldwide. Today, the fight against this disease relies heavily on the use of antimicrobial compounds, which have been widely used and prolonged use of antibiotics, 
Table 1. Frequency of Resistance of 178 E. coli Isolates From Broiler Chickens With Colibacillosis to 19 Antibiotics Tested

\begin{tabular}{|c|c|c|c|}
\hline $\begin{array}{l}\text { Antimicrobial } \\
\text { Agent }\end{array}$ & $\begin{array}{l}\text { Resistant } \\
\text { No. }(\%)\end{array}$ & $\begin{array}{l}\text { Intermediate } \\
\text { No. }(\%)\end{array}$ & $\begin{array}{l}\text { Sensitive } \\
\text { No. }(\%)\end{array}$ \\
\hline \multicolumn{4}{|l|}{ Macrolide groups } \\
\hline Tylosin & $173(97.20)$ & $5(2.80)$ & 0 \\
\hline \multicolumn{4}{|l|}{ Tetracycline groups } \\
\hline Oxytetracycline & $173(97.20)$ & $5(2.80)$ & 0 \\
\hline Chlortetracycline & $170(95.50)$ & $8(4.50)$ & 0 \\
\hline Doxycycline & $145(81.47)$ & $30(16.85)$ & $3(1.68)$ \\
\hline \multicolumn{4}{|l|}{ Fenicol groups } \\
\hline Chloramphenicol & $40(22.47)$ & $62(34.83)$ & $73(42.70)$ \\
\hline \multicolumn{4}{|c|}{ Quinolone and Fluoroquinolone groups } \\
\hline Flumequine & $175(98.31)$ & $3(1.69)$ & 0 \\
\hline Nalidixic acid & $174(97.75)$ & $4(2.25)$ & 0 \\
\hline Difloxacin & $159(89.32)$ & $11(6.18)$ & $8(4.50)$ \\
\hline Ciprofloxacin & $105(58.98)$ & $34(19.11)$ & $39(21.91)$ \\
\hline \multicolumn{4}{|c|}{ Aminoglycoside groups } \\
\hline Kanamycin & 105 (58.98) & $28(15.73)$ & $45(25.27)$ \\
\hline Gentamicin & $13(7.30)$ & $61(34.27)$ & $104(58.43)$ \\
\hline \multicolumn{4}{|l|}{ Lincosamide groups } \\
\hline Lincospectin & $65(36.52)$ & $40(22.47)$ & $73(41.01)$ \\
\hline \multicolumn{4}{|l|}{ Polymyxin groups } \\
\hline Colistin & $122(68.54)$ & $43(34.16)$ & $13(7.30)$ \\
\hline \multicolumn{4}{|l|}{ Penicillin groups } \\
\hline Amoxicillin & $124(69.67)$ & $48(26.96)$ & $4(2.25)$ \\
\hline \multicolumn{4}{|l|}{ Nitrofurazone groups } \\
\hline Furazolidone & $91(51.13)$ & $67(37.64)$ & $20(11.23)$ \\
\hline \multicolumn{4}{|l|}{ Sulfonamide groups } \\
\hline $\begin{array}{l}\text { Sulfamethoxazole+ } \\
\text { trimethoprim }\end{array}$ & $128(71.91)$ & $21(11.80)$ & $29(16.29)$ \\
\hline Fosbac & $9(5.05)$ & $49(27.53)$ & $120(67.42)$ \\
\hline \multicolumn{4}{|l|}{ Cephalosporin groups } \\
\hline Ceftriaxone & $7(3.93)$ & $10(5.62)$ & $161(90.54)$ \\
\hline Ceftazidime & 0 & 0 & $100(100.0)$ \\
\hline
\end{tabular}

leading to resistance to some bacterial strains, led to drug inefficiencies and treatment failure. ${ }^{6,9}$ Bacterial resistance to antibiotics is usually based on mechanisms such as the production of drug-degrading enzymes, changes in bacterial permeability to drugs, changes in drug receptors at the bacterial level, changes in bacterial cell wall structure, and access to sub-metabolic pathways which compensate for the drug-inhibited reaction, which is transmitted either from one bacterium to another through spontaneous mutation in genes that control bacterial susceptibility or through plasmid transferring ${ }^{1,10}$ In the present study, resistance to 10 antibiotics with common use in poultry industry (tylosin, amoxicillin, oxytetracycline, chlortetracycline, doxycycline, sulfamethoxazole + trimethoprim, flumequine, difloxacin, colistin and nalidixic acid) is high that 53\% of the antimicrobial compounds constituted. More than $95 \%$ of $E$. coli isolates were resistant to the five antibiotics (27\%) including flumequine, tylosin, oxytetracycline, chlortetracycline and nalidixic acid that the main reasons being non-normatively and prolonged use of these compounds, particularly refers to usage of tetracyclines and macrolides group in poultry farms as treatment and growth-promoting therapies. Other factors, such as the presence of resistance genes to antimicrobial drugs, as well as the ability to exchange resistance genes and their activity in different hosts, may play an important role in drug resistance. In this study, the resistance of the tested isolates to fluoroquinolones other than ciprofloxacin was almost similar to tetracyclines and macrolides. Percentage of low resistance to lincospectin, chloramphenicol, gentamicin, fuzbac and cephalosporins group compared to the drugs investigated in this study is due to limited use (due to the combination of structure and high cost) of these medicinal compounds in the prevention and treatment of commercial poultry flocks.

In recent decades, increasing resistance of $E$. coli poultry pathogens strains to antimicrobial compounds used in poultry industry has been reported, and the pattern of antibiotic resistance in various geophysical regions has been changing. ${ }^{13-15}$ Mellata et $\mathrm{al}^{5}$ examined the sensitivity of $101 \mathrm{E}$. coli isolates from poultry flocks in Algeria, although they showed the highest resistance to tetracycline, but resistance to sulfamethoxazole + trimethoprim, ampicillin and neomycin was at the next

Table 2. Multiple Resistance of 178 E. coli Isolates From Broiler Chickens With Colibacillosis

Against 19 Tested Antibiotics

\begin{tabular}{lc}
\hline Antibiotics No. & Resistant No. $(\%)$ \\
\hline 1 & $178(100.0)$ \\
2 & $178(100.0)$ \\
3 & $178(100.0)$ \\
4 & $172(96.6)$ \\
5 & $167(93.8)$ \\
6 & $160(89.8)$ \\
7 & $151(84.8)$ \\
8 & $145(81.4)$ \\
9 & $126(70.7)$ \\
10 & $113(63.4)$ \\
11 & $105(58.9)$ \\
12 & $84(47.2)$ \\
13 & $38(21.3)$ \\
14 & $25(14.0)$ \\
15 & $20(11.2)$ \\
16 & $11(6.1)$ \\
\hline 17 & $5(2.8)$ \\
\hline & $1(0.5)$ \\
\hline &
\end{tabular}


site.

Hanson et $\mathrm{al}^{16}$ in the study of $54 \mathrm{E}$. coli isolates in Thai broiler farms reported the highest resistance to tetracycline $(77.8 \%)$, fluorophenicol $(50 \%)$, ampicillin $(38.9 \%)$ and enrofloxacin (9.3\%), respectively. In the study of Shecho et $\mathrm{al}^{17}$ on a total of 14 antimicrobials included in the study panel, 0\%-96.15\% E. coli isolates were likely to express resistance to erythromycin, clindamycin, spectinomycin and ciprofloxacin. Multidrug resistance to more than two antibiotics was found in 24 $(92.30 \%)$ isolates. This study showed the high presence of antimicrobial resistant in isolates of E. coli. Zhao et $\mathrm{al}^{18}$, in a study of 95 APEC strains of E. coli in Georgia, also found the highest resistance to sulfamethoxazole (93\%) followed by tetracycline (87\%) and enrofloxacin (52\%). A study of 83 APEC strains of E. coli in Japan by Ozawa et $\mathrm{al}^{14}$ also reported the highest resistance to ampicillin (77.1\%) followed by oxytetracycline $(75.9 \%)$ and trimethoprim (25.3\%), Enrofloxacin (21.7\%) and fluorophenicol (0.6\%). According to Gregova and Kmet ${ }^{19}$ in Slovakia, the highest percentage of resistance of $\mathrm{E}$. coli isolates in slaughter poultry was related to ampicillin (89\%), enrofloxacin (43\%), tetracycline (33\%) and fluorophenicol (18\%). In a study by Khan et al, ${ }^{20} 30$ commercial poultry E. coli isolates in Bangladesh reported erythromycin and tetracycline resistance percentages of 100 and 93.3, respectively. Wakawa et $\mathrm{al}^{26}$ examined $67 \mathrm{E}$. coli isolates isolated from commercial poultry flocks with colibacillosis in Nigeria with the highest resistance to chloramphenicol (11.9\%) and tetracycline (10.5\%) and subsequently to them. Neomycin (5.9\%), sulfamethoxazole (4.5\%), enrofloxacin (0.3\%), ampicillin (2.9\%) and erythromycin (1.5\%) were observed. Jahantigh et al. ${ }^{22}$ In Sistan and Baluchestan province had the highest resistance of $E$. coli isolated from broiler herds with infected with colibacillosis against tetracycline (95.0\%) and ciprofloxacin (88.3\%), co-trimoxazole (86.7\%), lincospectin (52.5\%) and gentamicin $(21.7 \%)$ and also the prevalence of tet $A$, tet $B$, tet $C$ and tet $D$ genes of tetracycline resistance among 60 isolates were $96.7 \%, 38.3 \%, 7.731 \%$ and $8.30 \%$, respectively. Bakhshi et $\mathrm{al}^{23}$ In Yazd also reported the highest resistance to nalidixic acid (100\%) and ciprofloxacin (86\%) and the highest sensitivity to colistin (100\%) and gentamicin (93\%). Joshi et $\mathrm{al}^{8}$ reported that in $20 \mathrm{E}$. coli isolates collected from laying flocks involved with colibacillosis in India, the highest antibiotic resistance to cephalexin (73.68\%) and the lowest was related to chloramphenicol (0\%). Also, the percentages of resistance to enrofloxacin, neomycin, sulfamethoxazole, furazolidone and amikacin were $31.58,31.58,15.79,15.79$ and 5.26 , respectively. Sjölund et $\mathrm{al}^{24}$ examined 51 isolates obtained from poultry in Italy and found the highest resistance to ampicillin and nalidixic acid and the lowest in the cephalosporin group. Of the 51 isolates identified, 16 were APEC strains that possessed genes iucD, iss, cvi/cva, irp2 and papc. Ibrahim et $\mathrm{al}^{25}$ studied 269 APEC strains isolated from broiler herds in northern Jordan with the highest resistance related to sulfamethoxazole + trimethoprim (95.5\%), amoxicillin (93.3\%) and doxycycline (92.2\%). The lowest were ceftriaxone $(3.93 \%)$ and ceftazidime $(0 \%)$ and the frequencies of SitA, iss, iucD, iucC, ast A, tsh, cvi and irp2 genes were $97.4 \%, 93.3 \%, 75 \%, 74.00 \%, 71.00 \%, 46.5 \%$, $39.00 \%$ and $34.00 \%$, respectively.

In Iran, there have been several studies on the antibiotic resistance of $E$. coli in poultry, indicating that the drug resistance is varied and high in different parts of the country. Khoshkhoo and Peighambari ${ }^{21}$ examined 150 E. coli isolates from 30 broiler flocks in Tehran province showed resistance to difloxacin, sulfamethoxazole + trimethoprim, enrofloxacin, Linco-Spectin and neomycin were $84 \%, 72.6 \%, 66 \%, 764 \%$ and 52\% respectively.

In the study of Zahraei Salehi and Farashi Bonab ${ }^{4}$ on 50 APEC strains of $E$. coli isolated from broilers in Tabriz, the highest resistance was to two antibiotics erythromycin $(97 \%)$ and tetracycline (94\%). Firouzi et $\mathrm{al}^{32}$ study in $58 \mathrm{E}$. coli isolates around Shiraz showed that all isolates were resistant to tylosin and the resistance to doxycycline, oxytetracycline, enrofloxacin and chloramphenicol were $86.2 \%, 84.5 \%, 39 \%$ and $27.2 \%$, respectively. In the study of Saberfar et $\mathrm{al}^{15}$ in commercial farms throughout Iran showed the highest resistance to erythromycin (99\%) followed by tetracycline (96\%), neomycin (87\%), LincoSpectin (79\%), difloxacin (78\%), enrofloxacin (76\%), ampicillin (49\%) and fluorophenicol (39\%).

Azizpour and Saeidi Namin ${ }^{30}$ in Ardabil province showed the highest resistance of E. coli isolated from broilers with colibacillosis to tetracycline $(99.43 \%)$ and erythromycin (97.75\%) and the lowest to fluorophenicol (58.99\%) and lincospectin (36.52\%). Ghaniei and Peighambari $^{10}$ observed 943 E. coli isolates from 8 provinces (including Tehran, Mazandaran, Isfahan, Semnan, Golestan, Kurdistan, Kermanshah and Qazvin) that these isolates showed resistance 65.5\% to sulfamethoxazole + trimethoprim and $61.5 \%$ to doxycycline, $55.5 \%$ to colistin, $41.5 \%$ to enrofloxacin, and $34.5 \%$ to fluorophenicol. Seifi et $\mathrm{al}^{7}$ in Mazandaran broilers reported the highest percentage of resistance to tetracycline (71.25\%), erythromycin (65\%), ampicillin $(62.5 \%)$ and enrofloxacin (7.5\%), respectively.

The findings of the present study are somewhat consistent with those reported in other geophysical regions in terms of drug resistance ${ }^{15,20}$ but with the findings of some researchers are different. ${ }^{19}$ This discrepancy may be due to differences in the type, amount and persistence of antimicrobial compounds use, and temporal and spatial differences and differences in the isolates studied. Multiple drug resistance to antibiotic compounds is a major problem and a common phenomenon seen in the majority of $E$. coli strains of the poultry pathogen in which bacterial isolates are resistant to at least two types of antibiotics. ${ }^{26,27}$ Significant increases in the incidence of multiple resistance of $E$. coli isolates to antibiotic 
compounds over the last decade have been reported in many countries including $\operatorname{Iran}^{7,15}$ Germany, ${ }^{27}$ China, ${ }^{28}$ Bangladesh, ${ }^{29}$ India, ${ }^{20}$ Algeria, ${ }^{5}$ Nigeria, ${ }^{21}$ and Zimbabwe. ${ }^{16}$

In this study, all 178 isolates were resistant to three types of antibiotics and 145 isolates (81.14\%) to 8 types of antibiotics out of 19 tested antimicrobial compounds, mainly due to their continued use without careful evaluation of the drug. The level of bacterial susceptibility is in poultry flocks is alarm a threat to the transmission of resistance to humans. ${ }^{31}$ This is very serious in Iran and other developing countries, which do not abide the use of antibiotics and avoid consumption of meat and other animal products treated with antibiotics. The present study findings (that the isolates were resistant to more than three type of antibiotic) is consistent with the study Jahantigh et $\mathrm{al}^{22}$ of Saidi et $\mathrm{al}^{16}$ In the present study, the frequency of drug resistance of isolates belonging to a single poultry unit was varied.

\section{Conclusion}

Due to the variability of drug resistance pattern of isolates in farms, antimicrobial susceptibility test should be performed independently for each region or even each poultry unit prior to prescribing antibiotics to select the appropriate and effective drug for the treatment of poultry colibacillosis. The results of this study showed that the spectrum and resistance level of E. coli isolates were broad and high compared to the majority of antibiotics commonly used in Iranian poultry industry, which necessitated the implementation of a national monitoring plan for essential antimicrobial resistance seems necessary that prevents the spread of resistant bacteria and consequently increasing antibiotic resistance of the bacteria through the regular use of medicines in poultry farms, along with the promotion of hygiene and biosecurity. Based on the above study and similar results by other researchers, it can be concluded that the indiscriminate use of antibiotics causes the resistance of bacteria to antibiotics and resistance genes are transmitted more rapidly between pathogenic bacteria. Therefore, it is suggested that by studying the prevalence of resistance and transmission of pathogenic genes in bacteria, it is possible to prevent increasing in resistance of these antibiotics.

\section{Ethical Approval}

Not applicable.

\section{Conflict of Interest Disclosure}

The authors declare no conflict of interest.

\section{Authors' contributions}

AA: Supervision, concept, design, data gathering, analysis and interpretation of data, drafting of the manuscript, searching of databases, scientific revision of manuscript; CG: critical revision of the manuscript for important intellectual content.

\section{Acknowledgments}

The authors of the article are grateful for the contribution of Mohaghegh Ardabili University Vice -Chancellor of Research for assisting in this research.

\section{References}

1. Nolan LK, Barnes HJ, Vaillancourt JP, Abdul-Aziz T, Logue CM. Colibacillosis. In: Diseases of Poultry. Wiley; 2013:751-805.

2. Sciberras M, Pipová M, Regecová I, Jevinová P, Demjanová S. Antibiotic resistance of Escherichia coli isolated from broiler chickens. Folia Vet. 2019;63(3):1-8. doi:10.2478/fv2019-0021

3. Mbanga J, Nyararai YO. Virulence gene profiles of avian pathogenic Escherichia coli isolated from chickens with colibacillosis in Bulawayo, Zimbabwe. Onderstepoort J Vet Res. 2015;82(1):e1-e8. doi:10.4102/ojvr.v82i1.850

4. Zahraei Salehi T, Farashi Bonab S. Antibiotics susceptibility pattern of Escherichia coli strains isolated from chickens with colisepticemia in Tabriz province, Iran. Int J Poult Sci. 2006;5(7):677-684. doi:10.3923/ijps.2006.677.684

5. Mellata M, Bakour R, Jacquemin E, Mainil JG. Genotypic and phenotypic characterization of potential virulence of intestinal avian Escherichia coli strains isolated in Algeria. Avian Dis. 2001;45(3):670-679.

6. Amara A, Ziani Z, Bouzoubaa K. Antibioresistance of Escherichia coli strains isolated in Morocco from chickens with colibacillosis. Vet Microbiol. 1995;43(4):325-330. doi:10.1016/0378-1135(94)00101-2

7. Seifi S, Khoshbakht R, Atabak AR. Antibiotic susceptibility, serotyping and pathogenicity evaluation of avian Escherichia coli isolated from broilers in northern Iran. Bulg J Vet Med. 2015;18(2):173-179. doi:10.15547/bjvm.819

8. Joshi S, Singh R, Singh SP. Antibiotic resistance profile of Escherichia coli isolates from colibacillosis in and around Pantnagar, India. Vet World. 2012;5(7):405-408. doi:10.5455/vetworld.2012.405-408

9. Suresh T, Srinivasan D, Hatha AAM, Lakshmanaperumalsamy P. The incidence, antibiotic resistance and survival of Salmonella and Escherichia coli isolated from broiler chicken retail outlets. Microbes Environ. 2000;15(3):173-181. doi:10.1264/jsme2.2000.173

10. Ghaniei A, Peighambari SM. Antimicrobial susceptibility of one thousand bacterial isolates to five antibacterial agents commonly used in the Iranian poultry industry. Iranian J Vet Med. 2012; 6:1-5

11. Johnson JR, Kuskowski MA, Smith K, O'Bryan TT, Tatini S. Antimicrobial-resistant and extraintestinal pathogenic Escherichia coli in retail foods. J Infect Dis. 2005;191(7):1040-1049. doi:10.1086/428451

12. M100-S11, Performance standards for antimicrobial susceptibility testing. Clin Microbiol Newsl. 2001;23(6):49. doi:10.1016/s0196-4399(01)88009-0.

13. Raemdonck DL, Tanner AC, Tolling ST, Michener SL. In vitro susceptibility of avian Escherichia coli and Pasteurella multocida to danofloxacin and five other antimicrobials. Avian Dis. 1992;36(4):964-967. doi:10.2307/1591556

14. Ozawa M, Harada K, Kojima A, Asai T, Sameshima T. Antimicrobial susceptibilities, serogroups, and molecular characterization of avian pathogenic Escherichia coli isolates 
in Japan. Avian Dis. 2008;52(3):392-397. doi:10.1637/8193120907-Reg

15. Saberfar E, Pourakbari B, Chabokdavan K, Dolatshahi FT. Antimicrobial susceptibility of Escherichia coli isolated from Iranian broiler chicken flocks, 2005-2006. J Appl Poult Res. 2008;17(2):302-304. doi:10.3382/japr.2007-00102

16. Saidi B, Mafirakureva P, Mbanga J. Antimicrobial resistance of Escherichia coli isolated from chickens with colibacillosis in and around Harare, Zimbabwe. Avian Dis. 2013;57(1):152-154. doi:10.1637/10325-081512-Case.1

17. Shecho M, Thomas N, Kemal J, Muktar Y. Cloacael carriage and multidrug resistance Escherichia coli O157:H7 from poultry farms, eastern Ethiopia. J Vet Med. 2017;2017:8264583. doi:10.1155/2017/8264583

18. Zhao S, Maurer JJ, Hubert S, et al. Antimicrobial susceptibility and molecular characterization of avian pathogenic Escherichia coli isolates. Vet Microbiol. 2005;107(3-4):215-224. doi:10.1016/j.vetmic.2005.01.021

19. Gregova G, Kmet V. Antibiotic resistance and virulence of Escherichia coli strains isolated from animal rendering plant. Sci Rep. 2020;10(1):17108. doi:10.1038/s41598-02072851-5

20. Khan A, Das SC, Ramamurthy T, et al. Antibiotic resistance, virulence gene, and molecular profiles of Shiga toxinproducing Escherichia coli isolates from diverse sources in Calcutta, India. J Clin Microbiol. 2002;40(6):2009-2015. doi:10.1128/jcm.40.6.2009-2015.2002

21. Khoshkhoo PH, Peighambari SM. Determine the antimicrobial susceptibility and plasmid profiles of the Escherichia coli isolates from avian colibacillosis. J Vet Res. 2005; 60 (2): 95-105.

22. Jahantigh M, Samadi K, Esmaeelzadeh Dizaji R, Salari S. Antimicrobial resistance and prevalence of tetracycline resistance genes in Escherichia coli isolated from lesions of colibacillosis in broiler chickens in Sistan, Iran. BMC Vet Res. 2020;16(1):267. doi:10.1186/s12917-020-02488-z

23. Bakhshi M, Zandi H, Fatahi Bafghi M, Astani A, Ranjbar VR, Vakili M. A survey for phylogenetic relationship; presence of virulence genes and antibiotic resistance patterns of avian pathogenic and uropathogenic Escherichia coli isolated from poultry and humans in Yazd, Iran. Gene Rep. 2020;20:100725. doi:10.1016/j.genrep.2020.100725

24. Sjölund M, Bonnedahl J, Bengtsson S, Kahlmeter G.
P1021 A comparison of antimicrobial susceptibility in non-clinical and clinical isolates of Escherichia coli. Int J Antimicrob Agents. 2007(29):S273.

25. Ibrahim RA, Cryer TL, Lafi SQ, Basha EA, Good L, Tarazi YH. Identification of Escherichia coli from broiler chickens in Jordan, their antimicrobial resistance, gene characterization and the associated risk factors. BMC Vet Res. 2019;15(1):159. doi:10.1186/s12917-019-1901-1

26. Wakawa A, Mohammed F, Mamman H. Isolation and antibiotic susceptibility of Escherichia coli and Salmonella gallinarum isolated from rats in commercial poultry farms with recurrent colibacillosis and fowl typhoid cases in Zaria, Nigeria. J Vet Adv. 2015;5(11):1147-1152. doi:10.5455/jva.20151120021054

27. Guerra B, Junker E, Schroeter A, Malorny B, Lehmann S, Helmuth R. Phenotypic and genotypic characterization of antimicrobial resistance in German Escherichia coli isolates from cattle, swine and poultry. J Antimicrob Chemother. 2003;52(3):489-492. doi:10.1093/jac/dkg362

28. Li L, Jiang ZG, Xia LN, et al. Characterization of antimicrobial resistance and molecular determinants of beta-lactamase in Escherichia coli isolated from chickens in China during 1970-2007. Vet Microbiol. 2010;144(34):505-510. doi:10.1016/j.vetmic.2010.02.005

29. Rahman M, Rahman BM, Rahman B. Antibiogram and plasmid profile analysis of isolated Escherichia coli from broiler and layer. Res J Microbiol. 2008;3(2):82-90. doi:10.3923/jm.2008.82.90

30. Azizpour A, Saeidi Namin V. Investigation of antibiotic resistance patterns in Escherichia coli isolated from broiler chickens with colibacillosis to ten antibacterial agents commonly used in the Iranian poultry industry. J Compara Pathobiol Iran. 2017; 14 (4): 2345- 2352

31. Azizpour A. A survey on prevalence of Salmonella Enteritidis and Salmonella Typhimurium serotypes in broiler flocks of Ardabil province and determination of their antibiotics resistance to five antibacterial agents widely used in the Iranian medical field. J Health. 2018;9(2):143151. doi:10.29252/j.health.9.2.143

32. Firouzi R, Rajaian H, Daneshgar P. Antibiotic resistance of salmonella and Escherichia coli isolated from chicken in shiraz area. J Vet Res. 2008; 62 (6): 341-344. 